\title{
La « gamification » de la vie : sous couleur de jouer?
}

\section{Haydée Silva}

\section{(2) OpenEdition}

\section{Journals}

Édition électronique

URL : http://journals.openedition.org/sdj/261

DOI : $10.4000 /$ sdj.261

ISSN : 2269-2657

\section{Éditeur}

Laboratoire EXPERICE - Centre de Recherche Interuniversitaire Expérience Ressources Culturelles Education

\section{Référence électronique}

Haydée Silva, «La « gamification » de la vie : sous couleur de jouer? », Sciences du jeu [En ligne], 1 | 2013, mis en ligne le 01 octobre 2013, consulté le 27 mars 2021. URL : http:// journals.openedition.org/sdj/261 ; DOI : https://doi.org/10.4000/sdj.261

Ce document a été généré automatiquement le 27 mars 2021

\section{(c) (i) (9)}

La revue Sciences du jeu est mise à disposition selon les termes de la Licence Creative Commons Attribution - Pas d'Utilisation Commerciale - Pas de Modification 4.0 International. 


\title{
La « gamification » de la vie : sous couleur de jouer?
}

\author{
Haydée Silva
}

1 Depuis 2010, le mot « gamification » est dans le vent. Né à la croisée des jeux vidéo et du marketing, ce néologisme désigne aujourd'hui l'élargissement du paradigme ludique à des domaines dont il est censé être habituellement exclu : travail, santé, éducation... En tant que tel, il ouvre d'intéressantes perspectives de réflexion sur la place et la fonction de la notion de jeu dans la société actuelle.

2 En postulant que la gamification désigne « l'élargissement du paradigme ludique à des domaines dont il est censé être habituellement exclu ", nous choisissons délibérément un énoncé explicatif très général qui n'a pas de statut proprement définitionnel. En effet, pour aboutir à une définition du mot, entendue en tant que "proposition énonçant une équivalence entre un terme [...] et l'ensemble des termes connus qui l'explicitent » (Rey et Rey-Debove, 1996), il faudrait d'abord défricher un vaste corpus de définitions souvent complémentaires mais parfois contradictoires. ${ }^{1} \mathrm{Ce}$ chantier reste à ouvrir. Ici, nous souhaiterions simplement rappeler l'inutilité de mettre en concurrence ces différentes définitions afin de tenter de dégager la définition, celle qui fixerait une fois pour toutes le sens du mot. À nos yeux, suivant l'exemple donné par l'auteur de Sous couleur de jouer, il s'agit plutôt de faire jouer les écarts entre les définitions pour mieux éclairer la notion telle qu'elle se présente aujourd'hui « sous les espèces du nom et du verbe, dans le domaine du langage " (Henriot, 1983, p. 4). Autrement dit, sur la base des Investigations philosophiques de Ludwig Wittgenstein, au lieu de chercher le référent commun et originel à tout ce qu'on appelle « gamification ", il convient de chercher à démêler le « réseau complexe d'analogies [...] qui s'entrecroisent et s'enveloppent les unes aux autres » (Wittgenstein, 1961, p. 147, § 66) au sein de cette notion nouvelle mais dont les composantes ne sont pas neuves... Cette étape est importante dans la mesure où l'idée de gamification est non seulement une idée humaine mais aussi et peut-être surtout une idée de l'humain. 


\section{La gamification : une notion problématique et à problématiser}

3 La gamification apparaît aujourd'hui en général à ceux qui s'y intéressent sous l'aspect d'un mot mâtiné, qui non seulement combine un préfixe anglais et un suffixe français mais est souvent prononcé - en bon franglais - "gaimification». La dénomination, encore instable et peu construite depuis des postures scientifiques, est proche de bien d'autres: «ludification» (nos explorations semblent indiquer que le terme «ludification» est plus usité au Québec qu'en France); "pointification»; «funification» ou encore "ludicisation». Cette dernière appellation a l'avantage d'éviter le suffixe "-fication» (du latin facere, "faire »), qui renforce l'idée d'une transformation automatique et non problématique en jeu d'une activité habituellement exclue de la sphère ludique. En anglais, nous avons également relevé productivity games, surveillance entertainement, funware, playful design, behavioral games, game layer et applied gaming (cf. Deterding et alii, 2011a, p. 9). La note anecdotique est introduite par Robertson (2010), qui suggère une piste pour expliquer pourquoi on ne dit pas en anglais luding : cela ressemble trop à lewding (« obscénifier »).

Les considérations qui suivent peuvent s'appliquer à la plupart de ces termes qui gravitent autour de la notion vedette de "gamification ", même si chacun d'entre eux mériterait un développement à part.

5 Avant d'en venir à l'analyse de ce que la lecture des ouvrages de Jacques Henriot peut apporter à la compréhension de la gamification, arrêtons-nous brièvement sur ceux qui nous semblent être les quatre principaux achoppements de certains usages actuels de cette notion:

- La gamification n'est pas un phénomène nouveau.

- La notion de gamification reste étroitement liée au marketing.

- Parler de gamification a souvent des relents comportementalistes.

- Parler de gamification tend à promouvoir une vision réductrice du jeu.

\section{La gamification n'est pas un phénomène nouveau}

6 Une chose est sûre : les différentes manifestations de la réalité regroupées aujourd'hui sous le terme de "gamification" sont loin d'être un phénomène nouveau. En appliquant une ou plusieurs des définitions actuellement en cours, de nombreuses traces de gamification pourraient être identifiées au fil de l'histoire, à l'aide entre autres de Gilles Brougère (1995), Roger Caillois (1958), Alain Cotta (1980, 1993), Jean Duvignaud (1980), Johan Huizinga (1951), el Gamificator (2011), Jean-Michel Mehl (1990, 2010) ou Steffen Waltz (2011).

7 La nouveauté associée à la portée actuelle de la gamification tient moins au domaine du jeu proprement dit qu'à la dématérialisation des artefacts et à la massification des outils technologiques de numérisation :

La véritable révolution, vient de l'opportunité unique que présente le Web social et ouvert qui a émergé ces dernières années. Internet permet de gamifier plus facilement, plus rapidement, et en touchant toujours plus d'individus (Gamificator, 2011).

8 Ce lien entre technologies numériques et gamification a été exploré, entre autres, par Raessens (2006), Zimermann (2010) ou encore par la revue spécifiquement consacrée 
depuis 2001 à la recherche autour des jeux informatiques, Game Studies. The international journal of computer game research. Cependant, l'émergence du Web 2.0 ne suffit pas à expliquer l'intérêt croissant pour la gamification: le fort impact actuel de la notion tient notamment à son lien étroit avec un secteur d'activité particulier, celui du marketing.

\section{La gamification reste étroitement liée à un secteur économique d'activité}

9 En effet, quittant la sphère du monde enfantin qui a longtemps étayé le lien analogique de certains phénomènes avec le monde du jeu, la métaphore s'étend aujourd'hui à des domaines dont elle semblait exclue il y a encore un quart de siècle. Si l'on admet qu'étudier la gamification consiste à explorer de nouvelles logiques d'utilisation de la métaphore ludique destinées à légitimer certains usages sociaux à travers des valeurs symboliques données, il convient d'inscrire la réflexion dans une perspective à la fois diachronique et synchronique. Il faut aussi tenir compte des différents agents et acteurs impliqués. Or, pour l'instant, les acteurs les plus visibles (ou les plus audibles et « lisibles » sur la Toile) sont les promoteurs et les créateurs de la gamification dans le domaine du marketing; l'étude académique de la réception des dispositifs de gamification par les destinataires existe mais reste encore peu développée, d'autant plus que les ludologues ont probablement moins recours que les spécialistes de marketing à la nouvelle étiquette et se penchent sur le phénomène dans une optique plus large. ${ }^{2}$ Néanmoins, la portée de cet usage va bien au-delà des frontières du marketing. Ian Bogost écrit à ce sujet :

The rhetorical power of the word "gamification" is enormous, and it does precisely what the bullshitters want: it takes games -a mysterious, magical, powerful medium that has captured the attention of millions of people- and it makes them accessible in the context of contemporary business. [...] This rhetorical power derives from the "-ification" rather than from the "game". -ification involves simple, repeatable, proven techniques or devices: you can purify, beautify, falsify, terrify, and so forth (Bogost, 2011).

\section{Parler de gamification a souvent des relents comportementalistes}

10 À la lumière de ce qui précède, il n'est pas étonnant que certaines des définitions de la gamification soient ouvertement comportementalistes, telles celles proposées sur la brochure de l'agence marketing Bunchball :

At its core, gamification is all about statistics. [...] These statistics create another level to the game and motivate people to play more. In essence, the statistics become the game. [...] Gamification is a strategy for influencing and motivating the behavior of people (Bunchball, 2010, p. 4).

Ainsi, tel que le signale Mathieu Triclot :

La gamification, qui s'accompagne souvent d'un éloge du pouvoir des jeux et des joueurs, repose en réalité sur un mépris total du médium, réduit à des mécaniques pavloviennes. [...] les dispositifs gamifiés nous dépossèdent de notre puissance de décision, de notre capacité à agir sur le monde et ses cadres. Le monde n'est peutêtre pas tant en manque de games et de règles du jeu que de play, de comportements véritablement ludiques, de capacité à jouer avec les règles. [...] La critique laisse ouverte la possibilité d'un usage positif et subversif de la gamification (Triclot, 2011, p. 234). 
Cette dimension comportementaliste des usages dominants de la gamification est fondée sur une vision réductrice de ce que jeu veut dire.

\section{Parler de gamification promeut une vision réductrice du jeu}

Déjà en 1989, Jacques Henriot parmi d'autres signalait :

La nouveauté ne tient [...] pas à ce que l'on joue davantage, mais plus profondément, plus radicalement, à ce que l'idée même de Jeu se trouve prise pour modèle théorique, pour principe explicatif permettant de concevoir et d'interpréter un certain nombre de situations, d'en comprendre le sens, et peut-être de les dominer (Henriot, 1989, p. 32).

14 Les définitions de la gamification tendent pour l'instant à focaliser l'attention exclusivement sur un pan de la métaphore ludique, qui n'englobe ni le play ni le playing. À notre avis, choisir de forger " gamification » à partir de game et non de play revient à mettre implicitement l'accent sur les artefacts et les systèmes de dispositions sans y associer nécessairement la notion de jouant, c'est-à-dire de sujet qui joue mais surtout qui choisit de jouer. Michael Wu va même jusqu'à affirmer que c'est l'inconscience du joueur par rapport à son comportement qui constitue la gamification : " gamification refers to those applications in a non-game context, where players don't really know that they are actually playing a game » $(\mathrm{Wu}, 2011)$.

15 Ainsi, la plupart des usages actuels de la notion de gamification semble mettre l'accent sur les supports et les règles, tout en pariant sur le comportement attendu (engagement en anglais, implication...). Les idées de latitude, écart, recul - bref, de prise de distance - sont le plus souvent absentes, alors que c'est dans la tension et l'équilibre dynamique entre implication et distanciation que réside la possibilité de s'impliquer tout en étant critique, et donc d'être par exemple en mesure d'arrêter un jeu auquel on ne croit pas ou auquel on ne veut plus jouer.

En somme, la gamification semble fondée sur la croyance en la capacité d'un dispositif (matériel + règles) à induire un comportement et des états psychologiques donnés qui peut même être associée à une logique de propagande (Enkerli et Letellier, 2012). Le jeu Train créé en 2009 par Brenda Brathwaite pour mettre en évidence le risque de ne s'intéresser qu'à la mécanique du jeu est un exemple original de ce qui peut arriver lorsque l'attention est focalisée sur la règle et non sur le but à atteindre. En effet, « Le but du jeu est de mettre le plus rapidement et efficacement possible des pions de personnages dans des petits wagons. Le premier joueur à terminer tire une carte terminus pour révéler sa destination et sur ces cartes, le joueur lit Auschwitz ou Bergen-Belsen... » (Guillaud, 2012).

\section{La gamification à la lumière de la pensée de Jacques Henriot}

17 Pour une première approximation de la notion de gamification telle qu'elle se présente aujourd'hui, nous allons reprendre et retravailler ici quatre idées essentielles avancées par l'auteur de Sous couleur de jouer :

- Le statut nécessairement métaphorique et social des notions appartenant au réseau sémantique du jeu. 
- La distinction entre différents niveaux de cette métaphore.

- La dualité dynamique de l'attitude ludique.

- La distinction entre pensée, énonciation et acte ludiques.

\section{Le jeu comme métaphore et comme construction sociale}

D'emblée, Jacques Henriot affirme dans son second ouvrage consacré au jeu :

Dire qu'il y a jeu quelque part, dans le monde, en quelqu'un, ce n'est pas effectuer le constat de la présence effective d'une réalité qui serait observable et dont le sens tomberait sous le sens : c'est émettre une hypothèse, porter un jugement, appliquer au donné de l'expérience vécue une catégorie qui provient de la société où l'on vit et que véhicule la langue dont cette société fait l'instrument de sa culture (Henriot, 1989, p. 16).

19 C'est donc en reconnaissant le statut métaphorique et socialement construit de la notion hypercentrale de jeu et des notions périphériques qui gravitent autour d'elle, dont la gamification, qu'il est possible de les explorer en tant qu'objets culturels. Notre objectif n'est pas de nier la gamification, mais plutôt de mettre en évidence l'illusion d'un référent originel et unique derrière une telle étiquette. Autrement dit, croire que toute expression gamificatoire contient en elle-même toutes les caractéristiques associées au jeu revient à appliquer une logique non pas métaphorique mais métonymique (liant deux termes en raison de leur relation prétendument nécessaire) voire synecdochique (prenant la partie pour le tout), en ignorant que la notion de gamification n'est que l'une des fibres de la trame ludique de notre société.

Gilles Brougère $(1995,2005)$ a quant à lui souligné également à plusieurs reprises le besoin de comprendre à quelle logique d'interprétation renvoie la dénomination ludique, historiquement située et socialement construite.

\section{Les niveaux d'analyse de la métaphore ludique}

21 En 1969, Jacques Henriot proposait comme niveaux d'une définition provisoire de jeu ce à quoi joue celui qui joue (le système de règles), ce que fait celui qui joue (le jouer) et ce qui fait que l'on joue (l'activité d'un joueur jouant). Vingt ans plus tard, en s'attachant à dégager les nœuds du réseau sémantique lié au jeu, il a reformulé son modèle après avoir identifié trois acceptions principales de la métaphore de jeu: le matériel, la structure et la pratique ludiques. Gilles Brougère, dans Jeu et éducation (1995), a repris et redéfini ces trois catégories en en inversant l'ordre et en remplaçant la pratique par la situation. Nous avons retravaillé ce modèle quelques années plus tard (Silva, 1999), en classant les indices d'appartenance au réseau sémantique de la métaphore ludique en quatre grandes catégories : matériel, structures (au pluriel, pour distinguer différents types de systèmes, du constitutif au métaludique, en passant par le normatif et l'évolutif), contexte et attitude (ces deux dernières catégories permettant de rendre compte de la situation tout en séparant les indices extrinsèques au jeu et les indices relatifs à la subjectivité du joueur).

\begin{tabular}{|l|l|l|l|}
\hline Henriot, Le jeu (1969) & $\begin{array}{l}\text { Henriot, Sous couleur de } \\
\text { jouer (1989) }\end{array}$ & $\begin{array}{l}\text { Brougère, Jeu et } \\
\text { éducation (1995) }\end{array}$ & $\begin{array}{l}\text { Silva, Poétiques ludiques } \\
\text { (1999) }\end{array}$ \\
\hline
\end{tabular}




\begin{tabular}{|c|c|c|c|}
\hline $\begin{array}{l}\text { 1. Ce à quoi joue celui } \\
\text { qui joue } \\
\text { 2. Ce que fait celui } \\
\text { qui joue } \\
\text { 3. Ce qui fait que l'on } \\
\text { joue }\end{array}$ & $\begin{array}{l}\text { 1. Matériel ludique } \\
\text { 2. Structure ludique } \\
\text { 3. Pratique ludique }\end{array}$ & $\begin{array}{l}\text { Le jeu comme... } \\
\text { 1. situation } \\
\text { 2. structure } \\
\text { 3. matériel }\end{array}$ & $\begin{array}{l}\text { 1. Matériel ludique } \\
\text { a) Par convention } \\
\text { b) Potentiel } \\
\text { 2. Structures ludiques } \\
\text { a) Métaludiques } \\
\text { b) Constitutives } \\
\text { c) Normatives } \\
\text { d) Évolutives } \\
\text { 3. Contexte ludique } \\
\text { 4. Attitude ludique }\end{array}$ \\
\hline
\end{tabular}
actuellement privilégiées et de chercher à valider l'hypothèse selon laquelle les structures métaludiques et constitutives ont été majoritairement privilégiées devant les structures normatives et surtout devant les structures évolutives. Les structures métaludiques sont celles relatives à la conception d'un type de jeu; elles sont communes à différents jeux appartenant à une même famille. Les structures constitutives sont propres à un jeu spécifique; elle sont formulées et acquises de manière explicite. Les structures normatives correspondent à une maitrise pratique audelà d'une énonciation explicite; elles sont respectées par la plupart des «bons" joueurs. Les structures évolutives renvoient quant à elles aux actualisations singulières $\mathrm{du}$ jeu; ce sont elles qui régissent les règles effectivement appliquées au cours de la partie, dans la mesure où elles font appel à la capacité à utiliser de façon pertinente une infinité de combinaisons différentes (voir Silva, 1999, p. 263). Selon le type de structures privilégié, la métaphore ludique peut être simplificatrice - lorsqu'elle schématise la réalité à l'aide des trois premiers types de structures - ou complexificatrice, lorsqu'elle ouvre l'infini des possibles.

Il conviendrait également de se pencher sur les indices contextuels - liés aux variables socioculturelles, à la situation de réception et à l'interludicité -, bien moins homogènes que certains paraissent le croire. Les indices contextuels du jeu sont d'autant plus importants que la mention du contexte est récurrente dans les tentatives de définition de la gamification (cf. par exemple Deterding et alii, 2011a, p. 10 ; Gamificator, 2010 ; $\mathrm{Wu}, 2011)$, alors même que de nos jours les contextes ludiques se diversifient et se diluent suite à la dématérialisation des pratiques.

Bien d'autres problématiques encore mériteraient d'être relevées, relatives entre autres au lien entre gamification et indices ludiques; dans les limites de cet article, nous nous contenterons de noter le fait suivant : qu'il s'agisse de «ce qui fait que l'on joue " (Henriot, 1983), de pratique, de situation ou d'attitude, les quatre modèles mentionnés (Henriot, 1983 et 1989; Brougère, 1995; Silva, 1999) soulignent l'importance de prendre en compte le joueur en tant que sujet agissant. Or, ce sujet se caractérise non seulement par son action - il est de ce fait agent - mais aussi et surtout par sa disposition d'esprit par rapport à cette action - grâce à laquelle il devient acteur. 


\section{La tension et l'équilibre dynamique de l'attitude ludique} et mai 2012, le seul texte qui cite Jacques Henriot est celui de Mathieu Triclot. Ce dernier auteur résume ainsi l'apport du premier : «L'intérêt principal de l'argument d'Henriot est donc d'asseoir le primat de l'activité sur l'objet. L'activité "jouer" définit l'objet "jeu" » (Triclot, 2011, p. 24). En effet, Jacques Henriot souligne qu'il n'y a pas de matériel ni de structure qui soient intrinsèquement ludiques et en conclut que seule l'attitude ludique permet d'affirmer l'existence du jeu :

la seule "chose" qui soit à définir quand on parle de jeu est la forme de pensée, l'attitude mentale, la conscience singulière qui découvre dans ce matériel et cette structure des occasions ou des moyens de jouer (Henriot, 1989, p. 123).

Cette «importance croissante accordée au critère de l'attitude ludique semble s'inscrire dans une tendance générale à l'élargissement des intériorités personnelles et à une revendication du rôle des acteurs sociaux » (Silva, 1999, p. 272). Cette attitude est caractérisée, d'après Jacques Henriot, par l'association dynamique entre implication et distanciation (engagement/désengagement, rapprochement/recul). L'« intervalle qui sépare l'acteur de son acte fonde la duplicité du joueur qui sait qu'il joue.» (Henriot, 1983, pp. 82-83). Or, nous l'avons vu, la plupart des définitions actuelles de la gamification ne mettent l'accent que sur le premier des termes tout en omettant le second dans de nombreux cas. Cela accroît le risque de réification des agents du jeu, qui dès lors ne sont plus à même de profiter du potentiel heuristique du jeu. Or, le jeu n'est pas exclusivement constitué par les conditions formelles de son existence; il est également fondé sur l'acte qui les interprète: "jouer, c'est croire qu'on joue.» (Henriot, communication personnelle, cité dans Silva, 1999, p. 670).

\section{Qui pense, dit et/ou agit le jeu?}

À notre connaissance, Jacques Henriot n'a pas développé une piste fondamentale pourtant ébauchée dans Sous couleur de jouer : qui est le sujet de l'idée de jeu dans « une fois rassemblées les conditions formelles [...], il faut encore que l'idée vienne à quelqu'un de dire que c'est d'un jeu qu'il s'agit» (Henriot, 1989, p. 295; c'est nous qui soulignons)? S'agit-il forcément de celui qui joue, le sujet du jeu? Ou peut-il s'agir, comme nous le postulons, de celui qui attribue au jeu son sens de jeu, autrement dit, celui qui perçoit et interprète le jeu sans forcément y participer? En nous intéressant aux poétiques ludiques en critique et théorie littéraires, et en reprenant une distinction établie par Gilles Brougère entre "une reconnaissance objective par observation externe» et "un sentiment personnel que chacun peut avoir en certaines circonstances de participer à un jeu » (Brougère, 1995, p. 13), nous avions déjà tâché de montrer en quoi la distinction entre pensée, énonciation et acte ludiques nous semblait indispensable (Silva, 1999, pp. 166-172). À notre sens, cette distinction reste pertinente pour étudier aujourd'hui la portée de la gamification. C'est ce que nous allons désormais essayer d'expliquer à partir d'un exemple concret, celui de la gamification de l'éducation. 


\section{Que gagne-t-on à gamifier l'éducation ?}

29 Jeu et éducation entretiennent des rapports complexes depuis des temps très lointains (Brougère, 1995). Qu'apporte aujourd'hui la notion de gamification à ce binôme ancien? La gamification est-elle le moyen de rendre l'éducation plus attirante (selon une dialectique où jeu égale fun et travail égale ennui) ? S'agit-il de passer une couche de vernis supposément ludique sur une réalité censée être peu avenante ? À notre sens, dans la plupart des cas, la gamification n'apporte pour l'instant que les oripeaux des champs sociaux dont elle est issue (marketing et technologie, notamment); prise sans distance, elle peut conduire à une pure instrumentalisation du jeu au détriment des buts réels de l'apprentissage. Beaucoup de voix se sont d'ores et déjà élevées contre cette possibilité (voir par exemple Ely, 2011; Sierra, 2012; Enkerli dans Enkerli et Letellier, 2012).

30 La catégorie mentale « jeu » est parfois surimposée à des activités dont les agents sont loin de partager cette appréciation ludique. Tel que le signale olivier Mauco, l'importance donnée au score dans la gamification « réduit les pratiques à l'usage, et l'usage à la consommation.» (Mauco, 2012b). La pensée de jeu, suivie ou non d'énonciation, est ici dissociée de l'acte. Or, nous l'avons vu, la valeur de jeu n'est pas intrinsèque : elle est le produit du système dont elle fait partie et elle résulte du lien que l'être humain entretient aux objets dans ce système, c'est-à-dire de l'interprétation qui en est donnée. Le sens ne précède pas le sujet, il est à produire.

31 Le risque est grand de penser que la pensée du jeu suffit pour activer le potentiel heuristique de la métaphore de jeu; or, la capacité du jeu à ouvrir les possibles tient moins à l'acte ou à la pensée du jeu assumée et parfois explicitée par des tiers qu'à l'actualisation de l'attitude ludique par celui-là même qui joue. L'élargissement délibéré du paradigme ludique au domaine de l'éducation n'aura de sens que s'il est adopté de manière active par les acteurs de ce champ et associé à une vision critique, afin de laisser de côté les postulats explicites ou implicites hérités d'autres sphères d'activité pour ancrer la réflexion et l'action dans le monde de l'éducation. Autrement dit, il ne s'agit pas de "vendre » un projet pédagogique et de le surimposer aux agents éducatifs mais bien de le rendre plus efficace en transformant la disposition d'esprit des acteurs qui y participent.

Comme tous ceux ayant choisi de parcourir les sentiers infinis du jeu, nous avons la conviction qu'introduire le jeu et introduire $d u$ jeu dans différents domaines de la vie peut présenter certains avantages. En éducation, et probablement dans bien d'autres domaines, ces avantages semblent avant tout liés à la disposition intime du joueur, pleinement engagé dans son jeu tout en étant capable de prendre de la distance. Cette attitude ludique n'est pas compatible avec certains des avatars contemporains de la gamification, trop centrés sur la mécanique ou le dispositif ludiques au détriment du vécu subjectif du sujet qui leur donne sens et signification. Il serait illusoire de prétendre transformer l'éducation en jeu, baguette magique en main : on peut tout au plus faire basculer cette activité dans le champ métaphorique du jeu, vaste et contradictoire, en faisant augmenter son « coefficient de jouabilité » (Henriot, 1989, p. 217). Bref, une éducation gamifiée - ou, mieux, ludicisée - ne saurait être affaire de sens attribué en amont : elle est surtout affaire de signification en tant que production en aval. 


\section{BIBLIOGRAPHIE}

BOGOST I. (2011), « Gamification Is Bullshit », The Atlantic, 9 août 2011. Consulté en ligne le 12 avril 2012 :

http://www.theatlantic.com/technology/archive/2011/08/gamification-is-bullshit/243338/

BROUGERE G. (1995), Jeu et éducation, Paris, L'Harmattan.

BROUGERE G. (2005), Jouer/Apprendre, Paris, Anthropos.

Bunchball (2010), Gamification 101: An Introduction to the Use of Games Dynamics to Influence Behavior, San Jose, CA, Bunchball. Consulté en ligne le 20 avril 2012 :

http://www.bunchball.com/sites/default/files/downloads/gamification101.pdf

Bunchball (2011), Winning with Gamification. Tips from the Expert's Playbook, San Jose, CA, Bunchball. Consulté en ligne le 20 avril 2012 :

http://www.bunchball.com/sites/default/files/downloads/gamification-playbook.pdf

CAILLOIS R. (1958), Les Jeux et les hommes. Le Masque et le vertige, Paris, Gallimard.

COTTA A. (1980), La Société ludique. La Vie envahie par le jeu, Paris, Grasset.

COTTA A. (1993), La Société du jeu, Paris, Fayard.

DETERDING S., DIXON D., KHALED R., \& LENNART N. (2011a), « From Game Design Elements to Gamefulness: Defining “Gamification” ", MindTrek'11, pp. 9-15.

DETERDING S., SICART M., LENNART N., O'HARA K., \& DIXON D. (2011b), « Gamification. Using Game-Design Elements in Non-Gaming Contexts », Proceedings of CHI 2011 Workshop Gamification. Consulté en ligne le 13 avril 2012 :

http://gamification-research.org/wp-content/uploads/2011/04/01-Deterding-Sicart-NackeOHara-Dixon.pdf

DUVIGNAUD J. (1980), Le Jeu du jeu, Paris, Balland.

ELY M. (2011), « 3 Reasons NOT to Gamify Education », LearnBoost Blog, $1^{\text {er }}$ septembre 2011. Consulté en ligne le 21 avril 2012 :

Sciences du jeu, 1 | 2013 
https://www.learnboost.com/blog/blog/3-reasons-not-to-gamify-education/

ENKERLI A. et LETELLIER S. (2012), « La ludification, un concept à la mode », La Sphère / entretien radiophonique, 31 mars 2012. Consulté en ligne le 20 avril 2012 :

http://www.radio-canada.ca/audio-video/pop.shtml\#urlMedia=http://www.radio-canada.ca/ Medianet/2012/CBF/LaSphere201203311605_1.asX

FINDLAY K. \& ALBERTS K. (2011), « Gamification. How effective is it ? », Slideshare, 26 septembre 2011. Consulté en ligne le 21 avril 2012 :

http://www.slideshare.net/ervler/gamification-how-effective-is-it

Gamificator (2011), La gamification pour les nuls / blogue, 2011. Consulté en ligne le 14 avril 2012 :

http://www.elgamificator.com/gamification

GUILLAUD H. (2012), « Comment le jeu code-t-il le monde ? », Internet actu.net, 8 mars 2012.

Consulté en ligne le 14 avril 2012 :

http://www.internetactu.net/2012/03/08/lift12-comment-le-jeu-code-t-il-le-monde/

HENRIOT J. (1983), Le Jeu [1969], Paris, Synonyme SOR.

HENRIOT J. (1989), Sous couleur de jouer. La Métaphore ludique, Paris, José Corti.

HUIZINGA J. (1951), Homo ludens. Essai sur la fonction sociale du jeu [1938], Paris, Gallimard.

HUOTARI H. \& HAMARI J. (2011), « "Gamification” from the perspective of service marketing », Proceedings of CHI 2011 Workshop Gamification. Consulté en ligne le 21 avril 2012 :

http://gamification-research.org/wp-content/uploads/2011/04/14-Huotari.pdf

KIM A.J. (2010), « Gamification Workshop Presentation » / diaporama, Gamification wiki. Consulté en ligne le 12 avril 2012 :

http://gamification.org/wiki/Amy_Jo_Kim_Gamification_Workshop

LE NAGARD E. (2012), « Jouer n'est-il qu'un jeu ? Quelques pistes de réflexion autour de la gamification », blogue d'Erwan Le Nagard, 5 février 2012. Consulté en ligne le 14 avril 2012 :

http://www.erwanlenagard.com/rebonds/jouer-nest-il-quun-jeu-quelques-pistes-de-reflexionautour-de-la-gamification-552

MACCLELLAND C. (2010), « Engagement through Gamification Presentation » / diaporama. Gamification wiki. Consulté en ligne le 12 avril 2012 :

http://gamification.org/wiki/Engagement_through_Gamification

MAUCO O. (2012a), « Repenser la gamification » / diaporama, Game in Society, 7 mars 2012. Consulté en ligne le 14 avril 2012 :

http://www.gameinsociety.com/post/2012/03/07/Intervention-sur-la-gamification-les-slides

MAUCO O. (2012b), « Sur la gamification », Game in Society, 19 janvier 2012. Consulté en ligne le 14 avril 2012 :

http://www.gameinsociety.com/public/Sur_la_gamification_-_Mauco__gameinsociety.pdf MEHL J-M. (1990), Les Jeux au royaume de France du XIIIe au début du XVIe siècle, Paris, Fayard. MEHL J-M. (2010), Des jeux et des hommes dans la société médiévale, Paris, Honoré Champion. REY A. et REY-DEBOVE J. (dir.) (1996), Le Nouveau Petit Robert. Dictionnaire alphabétique et analogique de la langue française / version électronique sur CD-ROM. Paris, Dictionnaires Le Robert. 
ROBERT T. et AYERDI C. (2011), « La ludification de l'univers, rien de moins », Argus Montréal. 40.1, pp. 24-27.

SIERRA K. (2012), « Kathy Sierra. On Gamification In Education », Larry Ferlazzo's Websites of the Day / blogue, 26 février 2012. Consulté en ligne le 21 avril 2012 :

http://larryferlazzo.edublogs.org/2012/02/26/kathy-sierra-on-gamification-in-education/

SILVA H. (1999), Poétiques du jeu. La métaphore ludique dans la théorie et la critique littéraires françaises au XXe siècle, thèse de doctorat, Université Paris III.

TRICLOT M. (2011), Philosophie des jeux vidéo, Paris, Zones.

WALLACE M. (s.d.), « The Gamification of Everything. Presentation » / diaporama, Gamification wiki. Consulté en ligne le 12 avril 2012 :

http://gamification.org/wiki/The_Gamification_of_Everything

WALTZ S. (2011), « The Lowdown On Gamification. Or: a Gamificationization » / diaporama, Slideshare, 3 février 2011. Consulté en ligne le 21 avril 2012 :

http://www.slideshare.net/playbe/steffenpwalzlift11110203

WU M. (2011), « What is Gamification, Really? », Lithium Lithosphere. 29 août 2011. Consulté en ligne le 12 avril 2012 :

http://lithosphere.lithium.com/t5/Building-Community-the-Platform/What-is-GamificationReally/ba-p/30447

\section{NOTES}

1. Pour des définitions en anglais, voir entre autres Bunchball, 2010, p. 2 ; Bunchball, 2011, p. 2 ; Deterding et alii, 2011a, pp. 9-10 ; Deterding et alii, 2011b, p. 1 ; Findlay \& Alberts, 2011 ; Huotari \& Hamari, 2011 ; Kim, 2010, pp. 4-7 ; MacClelland, 2010, p. 2 ; Wallace, s.d., pp. 5-6 ; Wu, 2011. Pour des définitions en français, consulter par exemple Gamificator, 2011 ; Guillaud, 2012 ; Le Nagard, 2012 ; Mauco, 2012a, p. 11 ; Mauco, 2012b ; Robert et Ayerdi, 2011.

2. Ainsi, dans les 11 volumes et 17 numéros de Game Studies, on ne retrouve aucune occurrence de "gamification» ou de "ludification» jusqu'en décembre 2011. Sébastien Genvo (voir sa contribution à ce numéro) recourt quant à lui au terme de "ludicisation ». Une recherche effectuée en juin 2012 sur le moteur de recherche Google à partir de ce dernier mot clé renvoyait à 526 occurrences à peine, contre 884000 pour " gamification »; en avril 2013, «ludicisation » atteignait déjà 12100 résultats, contre 1650000 pour le terme concurrent.

\section{RÉSUMÉS}

Le mot « gamification » a aujourd'hui le vent en poupe et désigne l'élargissement du paradigme ludique à des domaines dont il est censé être habituellement exclu. S'il ouvre d'intéressantes perspectives de recherche sur la place et la fonction de la notion de jeu dans la société actuelle, il doit aussi être problématisé. Après avoir évoqué ceux qui nous semblent être les quatre 
principaux achoppements de certains usages actuels de cette notion, nous explorerons la gamification à la lumière de la pensée de Jacques Henriot. Nous reprendrons notamment des éléments de discussion autour du jeu comme métaphore et comme construction sociale, des niveaux d'analyse de la métaphore ludique, de la tension et l'équilibre dynamique de l'attitude ludique, ainsi que de la différence entre pensée, énonciation et action. Finalement, nous esquisserons des pistes de réflexion pour aborder lees liens entre gamification et éducation.

"Gamification » is now a buzzword ; it denotes the widening of the ludic paradigm to fields from which it is supposed to be usually excluded. The notion opens up interesting research perspectives about the place and the function of game's and play's notions in today's society, but it must also be problematized. After referring to those that we think should be seen as four main obstacles for certain current uses of this notion, we explore gamification based of Jacques Henriot's thought. We will include discussion elements around game and play as metaphors and as social constructs, levels of analysis of the playful metaphor, tension and dynamic balance of playfulness and difference between thinking, saying and acting. Finally, we shall sketch some guidelines to approach the links between gamification and education.

INDEX

Keywords : gamification, Henriot, metaphor, playful attitude, education

Mots-clés : gamification, Henriot, métaphore, attitude ludique, éducation

\section{AUTEUR}

\section{HAYDÉE SILVA}

Universidad Nacional Autónoma de México 